\title{
Electroweak and non-resonant corrections to top-pair production near threshold at NNLO
}

\section{Martin Beneke}

Physik Department T31, James-Franck-Straße 1, Technische Universität München, 85748 Garching, Germany

\section{Andreas Maier}

IPPP, Department of Physics, University of Durham, DH1 3LE, United Kingdom

Thomas Rauh*i

IPPP, Department of Physics, University of Durham, DH1 3LE, United Kingdom

E-mail: thomas.j.rauh@durham.ac.uk

\section{Pedro Ruiz-Femenía}

Departamento de Física Teórica and Instituto de Física Teórica UAM-CSIC,

Universidad Autónoma de Madrid, E-28049 Madrid, Spain

The top-quark mass can be determined with very high precision from a scan of the total $e^{+} e^{-} \rightarrow$ $b \bar{b} W^{+} W^{-} X$ cross section near the top-pair production threshold. We present the full calculation of electroweak and non-resonant corrections to this process at NNLO. We discuss the size of the new contributions and estimate the theory uncertainty on the top-quark mass in the PS mass scheme.

13th International Symposium on Radiative Corrections (Applications of Quantum Field Theory to Phenomenology)

25-29 September, 2017

St. Gilgen, Austria

\footnotetext{
*Speaker.

${ }^{\dagger}$ TUM-HEP-1105/17, IPPP/17/81, FTUAM-17-25, IFT-UAM/CSIC-17-106
} 


\section{Introduction}

The total inclusive cross section for the process $e^{+} e^{-} \rightarrow b \bar{b} W^{+} W^{-} X$ is highly sensitive to the value of the top-quark mass in the vicinity of the top-pair production threshold $\sqrt{s}=2 m_{t}$. At a future lepton collider this facilitates the determination of the top-quark mass in a well-defined mass scheme like $\overline{\mathrm{MS}}$ or PS [1] with an uncertainty of about $50 \mathrm{MeV}$ [2, 3]. In addition the strong coupling constant and the top-quark width and Yukawa coupling can be determined. Given that theoretical uncertainties dominate over statistical ones, precise predictions for the cross section are crucial for this programme.

The challenge in higher-order computations in the threshold region lies in dealing with the non-relativistic nature of the process. The non-relativistic top pair interacts strongly through a non-local color Coulomb potential which manifests itself at the level of the cross section as corrections scaling with powers of $\alpha_{s} / v$, where $v$ is the top-quark velocity. These effects become non-perturbatively strong in the threshold region, where $v \sim \alpha_{s}$, and must be resummed. The techniques to perform this resummation are based on effective field theories (EFT) and exploit the hierarchy between the dynamical scales $m_{t}$ (hard), $m_{t} v$ (soft) and $m_{t} v^{2}$ (ultrasoft) of the process. By integrating out the hard and soft modes the problem of solving multiscale Feynman integrals is divided into simpler calculations of matching coefficients and of a non-relativistic Green function. Within this approach the QCD corrections have been computed up to NNNLO [3], where the EFT framework is given by potential non-relativistic QCD (PNRQCD) [4, 5].

The scale uncertainty of the NNNLO QCD result is at the level of only $\pm 3 \%$. We find, however, that non-QCD effects at NLO yield corrections up to $15 \%[6,7]$, which motivates the calculation of the full NNLO non-QCD corrections [8] presented in these proceedings. The extension of the calculation beyond pure QCD requires a systematic treatment of the instability of the top quark. Counting $\alpha_{\mathrm{EW}} \sim \alpha_{s}^{2} \sim v^{2}$, the top-quark decay width $\Gamma_{t} \sim m_{t} \alpha_{\mathrm{EW}}$ is of the same order as the ultrasoft scale $m_{t} v^{2}$, which implies that the narrow width approximation for the top quarks is unphysical and that we must consider the final state $b \bar{b} W^{+} W^{-} X$ after top decay. Consequently, we have to take into account not only the resonant production of the final state through the decay of an intermediate non-relativistic top pair, but also the non-resonant production through hard processes as shown in Figure 1. The example diagrams are of the relative order $\alpha_{\mathrm{EW}} / v \sim \alpha_{s}$, where the $1 / v$ factor accounts for the phase-space suppression for the production of a non-relativistic particle pair in the resonant part which is not present in the non-resonant contribution, and therefore contribute at NLO.
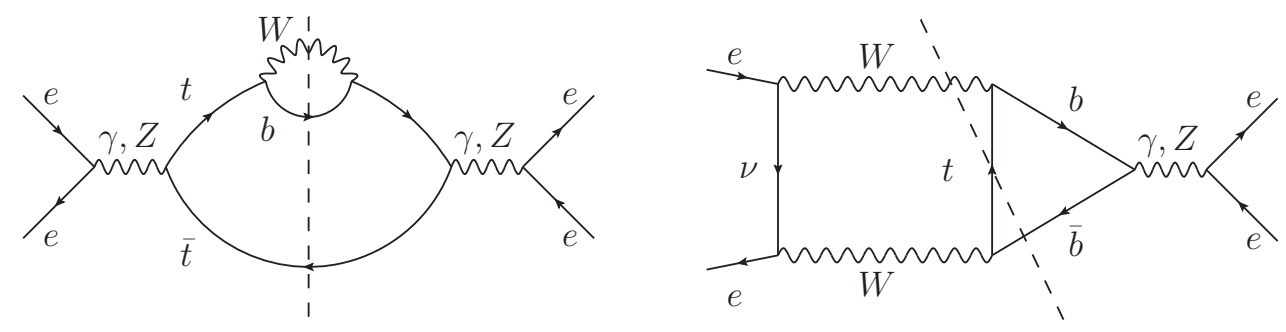

Figure 1: Example diagrams contributing to the non-resonant cross section at NLO. In the left diagram, the top lines are off-shell, and the antitop ones near mass-shell. 
For the systematic calculation of the non-QCD effects the EFT framework must be extended to Unstable Particle Effective Theory [9, 10]. Utilizing the optical theorem, the cross section takes the form

$$
\begin{aligned}
\sigma(s) \sim \operatorname{Im} & {\left[\sum_{k, l} C^{(k)} C^{(l)} \int d^{4} x\left\langle e^{-} e^{+}\left|\mathrm{T}\left[i \mathscr{O}^{(k) \dagger}(0) i \mathscr{O}^{(l)}(x)\right]\right| e^{-} e^{+}\right\rangle\right.} \\
& \left.+\sum_{k} C_{4 e}^{(k)}\left\langle e^{-} e^{+}\left|i \mathscr{O}_{4 e}^{(k)}(0)\right| e^{-} e^{+}\right\rangle\right]
\end{aligned}
$$

where only cuts corresponding to the final state $b \bar{b} W W X$ must be considered when taking the imaginary part. The first line of (1.1) corresponds to the resonant cross section, where the production operator $\mathscr{O}^{(l)}$ annihilates the external $e^{+} e^{-}$state and produces a non-relativistic top pair. The hard matching coefficients $C^{(l)}$ absorb the corrections to the production from hard modes. The matrix element must be evaluated with the EFT given by the Lagrangian

$$
\mathscr{L}=\mathscr{L}_{\mathrm{PNREFT}}+\mathscr{L}_{\mathrm{SCET}}^{(-)}+\mathscr{L}_{\mathrm{SCET}}^{(+)}
$$

where $\mathscr{L}_{\text {PNREFT }}$ follows from the generalization of PNRQCD to electroweak effects and contains the interactions of the non-relativistic top quarks through potentials and with ultrasoft modes and $\mathscr{L}_{\text {SCET }}^{(\mp)}$ contains the interactions of the collinear electron and positron with collinear and ultrasoft modes. ${ }^{1}$ There are no interactions of the top quarks with collinear modes in the EFT, because momentum conservation implies that they create hard modes which have been integrated out.

The second line of (1.1) corresponds to the non-resonant contribution, which is given by the matrix elements of local four-electron operators since the non-resonant production is a hard process and the hard scale has been integrated out in the EFT. The hard matching coefficients $C_{4 e}^{(k)}$ contain imaginary parts from cuts corresponding to the $b \bar{b} W^{+} W^{-} X$ final state. In practice, we do not determine the coefficients, but compute the non-resonant cross section directly. The full NLO contribution is given by the sum of the cross sections for the processes $e^{+} e^{-} \rightarrow \bar{t} b W^{+}$and $e^{+} e^{-} \rightarrow$ $t \bar{b} W^{-}$evaluated exactly at the threshold $s=4 m_{t}^{2}$ and has been computed in [6]. Setting $s=4 m_{t}^{2}$ isolates the contribution from the hard momentum region, since all other modes only yield scaleless integrals that vanish in dimensional regularization. The NNLO corrections are given by the $\mathscr{O}\left(\alpha_{s}\right)$ corrections to these processes. Doubly non-resonant contributions scale as $\alpha_{\mathrm{EW}}^{2} / v \sim \alpha_{s}^{3}$ relative to the LO and need not be considered at NNLO.

The resonant and non-resonant contributions contain finite-width and endpoint divergences, respectively, which cancel in the sum (1.1) over both parts. Such spurious divergences are common when momentum regions are separated [11, 12]. In the resonant part, finite-width divergences appear in the UV region of the loop integration over the momenta of the non-relativistic top quarks, i.e. in the limit where top quarks that are parametrically close to resonance are far off-shell. They have been regularized dimensionally and we must employ the same scheme for the computation of the non-resonant part. The origin of the divergences in the non-resonant part is discussed below.

\footnotetext{
${ }^{1}$ It is also possible to integrate out generic collinear modes, keeping only external-collinear modes whose momentum differs from that of the external electron or positron by an ultrasoft amount, see $[9,10]$.
} 


\section{Non-resonant part at NNLO}

As discussed above the non-resonant contribution up the NNLO is given by the sum of the processes $e^{+} e^{-} \rightarrow \bar{t} b W^{+}$and $e^{+} e^{-} \rightarrow t \bar{b} W^{-}$at threshold at NLO in QCD. This is not a standard $2 \rightarrow 3$ NLO calculation because of the presence of endpoint divergences which must be regularized dimensionally. To illustrate this issue we consider the phase-space integral of one of the tree level diagrams

$$
\begin{aligned}
& \int d \operatorname{LIPS}_{e^{+} e^{-} \rightarrow \bar{t} b W^{+}} f_{i}\left(p_{e^{+}}, p_{e^{-}}, p_{\bar{t}}, p_{W^{+}}, p_{b}\right) \\
= & \frac{m_{t}^{2}}{2 \pi} \int_{x}^{1} d t \int d \operatorname{LIPS}_{e^{+} e^{-} \rightarrow t^{*} \bar{t}} \int d \operatorname{LIPS}_{t^{*} \rightarrow b W^{+}} f_{i}\left(p_{e^{+}}, p_{e^{-}}, p_{\bar{t}}, p_{W^{+}}, p_{b}\right) \\
\equiv & \int_{x}^{1} d t g_{i}(t),
\end{aligned}
$$

where standard phase-space integration techniques have been applied to split the $2 \rightarrow 3$ process into $2 \rightarrow 1+1^{*}$ and $1^{*} \rightarrow 2$ parts with an additional integration over the invariant mass $t=p_{t^{*}}^{2} / m_{t}^{2}$ of the off-shell top quark. The integration limits $x=m_{W}^{2} / m_{t}^{2}$ and 1 follow from the kinematic restrictions when the bottom-quark mass is neglected. The divergences originate from the endpoint $t \rightarrow 1$ of the integration, where the top-quark, which is parametrically off-shell, becomes resonant. This is exactly the opposite of the scenario in which the finite-width divergences occur in the resonant part. E.g. the integrand of the left diagram in Figure 1 takes the form

$$
g_{h_{1}}(t) \stackrel{t \rightarrow 1}{\propto} \frac{(1-t)^{1 / 2-\varepsilon}}{(1-t)^{2}}
$$

where the numerator is a phase-space suppression factor and the denominator comes from the two top-quark propagators. We note that the width-dependent term in the denominator $\left(p_{t^{*}}^{2}-m_{t}^{2}+\right.$ $i m_{t} \Gamma_{t}$ ) of the top propagator has been expanded out in (2.2) because in the hard momentum region $p_{t^{*}}^{2}-m_{t}^{2} \sim m_{t}^{2}$ is parametrically much larger than $m_{t} \Gamma_{t} \sim m_{t}^{2} \alpha_{\mathrm{EW}}$. The contribution from this diagram is divergent but finite in dimensional regularization, because the endpoint divergence as $t \rightarrow 1$ is non-logarithmic. The other tree-level diagrams contain at most one top-quark propagator and are finite [6], because there are only integrable divergences as $t \rightarrow 1$.

At NNLO this holds no longer true and explicit $1 / \varepsilon$ poles appear. We cannot expand the integrands $g_{i}$ of the NNLO diagrams in $\varepsilon$ without spoiling the dimensional regularization of the remaining $t$-integration. However, the computation of the full $\varepsilon$-dependence of the $g_{i}$, which contain phase-space and (for the virtual corrections) loop integrations, is difficult. We therefore use subtractions for the endpoint divergences

$$
\int_{x}^{1} d t g_{i}(t)=\int_{x}^{1} d t\left[g_{i}(t)-\sum_{a=1, \frac{3}{2}, 2} \sum_{b} \frac{\hat{g}_{i}^{(a, b)}}{(1-t)^{a+b \varepsilon}}\right]+\sum_{a=1, \frac{3}{2}, 2} \sum_{b} \frac{\hat{g}_{i}^{(a, b)}(1-x)^{1-a-b \varepsilon}}{1-a-b \varepsilon}
$$

where the expression in square brackets is finite and can be expanded in $\varepsilon$ before the integration over $t$ and the second term is the integrated subtraction term. The subtraction terms are given by the singular terms in $(1-t)$ with the full $\varepsilon$ dependence and have been determined in [13,8] using the expansion by regions $[11,12]$. The NNLO contributions contain endpoint singular terms scaling as 
$(1-t)^{-(a+b \varepsilon)}$ with $a=2,3 / 2,1$. Only those with $a=1$ yield explicit poles in $\varepsilon$, whereas the other terms are divergent but finite in dimensional regularization.

We compute the contributions from the endpoint divergent diagrams manually using the subtractions (2.3). For the endpoint finite diagrams we use MadGraph [14] code from which we remove the contributions from the endpoint divergent diagrams. The details of this computation are given in [8].

\section{Electroweak corrections to the resonant part at NNLO}

The resonant part receives corrections due to the decays of the top quarks and other electroweak contributions. At NNLO, we have to consider $\mathscr{O}\left(\alpha_{\mathrm{EW}}\right)$ corrections to the hard matching coefficients $C^{(k)}$ [15], which become complex because of $\bar{t} b W^{+}$and $t \bar{b} W^{-}$cuts [16, 8]. Furthermore, there are corrections to the matrix element from the QED Coulomb potential $-e_{t}^{2} \alpha_{\mathrm{em}} / r$ and higher-order terms involving the top-quark width, e.g. time dilatation effects. Last but not least, we have to consider photon radiation from the initial state (ISR). The ISR yields large logarithms $\ln \left(m_{e}^{2} / s\right)$. Thus, in addition to the fixed order $\mathscr{O}\left(\alpha_{\mathrm{EW}}\right)$ ISR corrections required at NNLO, we resum logarithms at the LL level using the structure function approach where the cross section takes the form

$$
\sigma_{\mathrm{w} . \mathrm{ISR}}(s)=\int_{0}^{1} d x_{1} \int_{0}^{1} d x_{2} \Gamma_{e e}^{\mathrm{LL}}\left(x_{1}\right) \Gamma_{e e}^{\mathrm{LL}}\left(x_{2}\right) \sigma\left(x_{1} x_{2} s\right) .
$$

The structure functions $\Gamma_{e e}^{\mathrm{LL}}(x)$ give the probability of finding an electron with momentum $x p$ in the 'parent electron' with momentum $p$ and can be found in [17]. They are currently not known at NLL. A detailed discussion of the corrections to the resonant part is given in [8].

\section{Phenomenology}

The new results have been added to a new version of the QQbar_Threshold code [18], which will soon become public. The effect of the non-QCD corrections is shown in Figure 2. The input values are $m_{t}^{\mathrm{PS}}=171.5 \mathrm{GeV}, \Gamma_{t}=1.33 \mathrm{GeV}$ and the default values of the code for all other parameters. Our central scale choices are $\mu_{r}=80 \mathrm{GeV}$ and $\mu_{w}=350 \mathrm{GeV}$ where $\mu_{r}$ is the renormalization scale and $\mu_{w}$ is the scale associated with the finite-width divergences. The dependence on the latter cancels exactly at NNLO when all corrections are included and only a tiny dependence remains at NNNLO where not all contributions are known. The QCD cross section is the sum of the S-wave [3] and P-wave [19] contributions while the full results includes in addition all NNLO corrections [8] and Higgs effects at NNNLO [7], but no ISR effects. The bands in Figure 2 are spanned by variation of the renormalization scale between 50 and $350 \mathrm{GeV}$. The largest corrections are observed below the threshold where the cross section is reduced by up to $25 \%$. This is where the cross section itself becomes small and the almost energy-independent non-resonant contribution yields a large relative correction. The non-QCD corrections also modify the shape of the cross section, making the remnant of the $1 \mathrm{~S}$ toponium peak more pronounced.

The ISR corrections are shown in Figure 3 and reduce the cross section by $30-45 \%$. The peak is smeared out and shifted to the right by almost $200 \mathrm{MeV}$. This emphasizes the need for a full NLL 

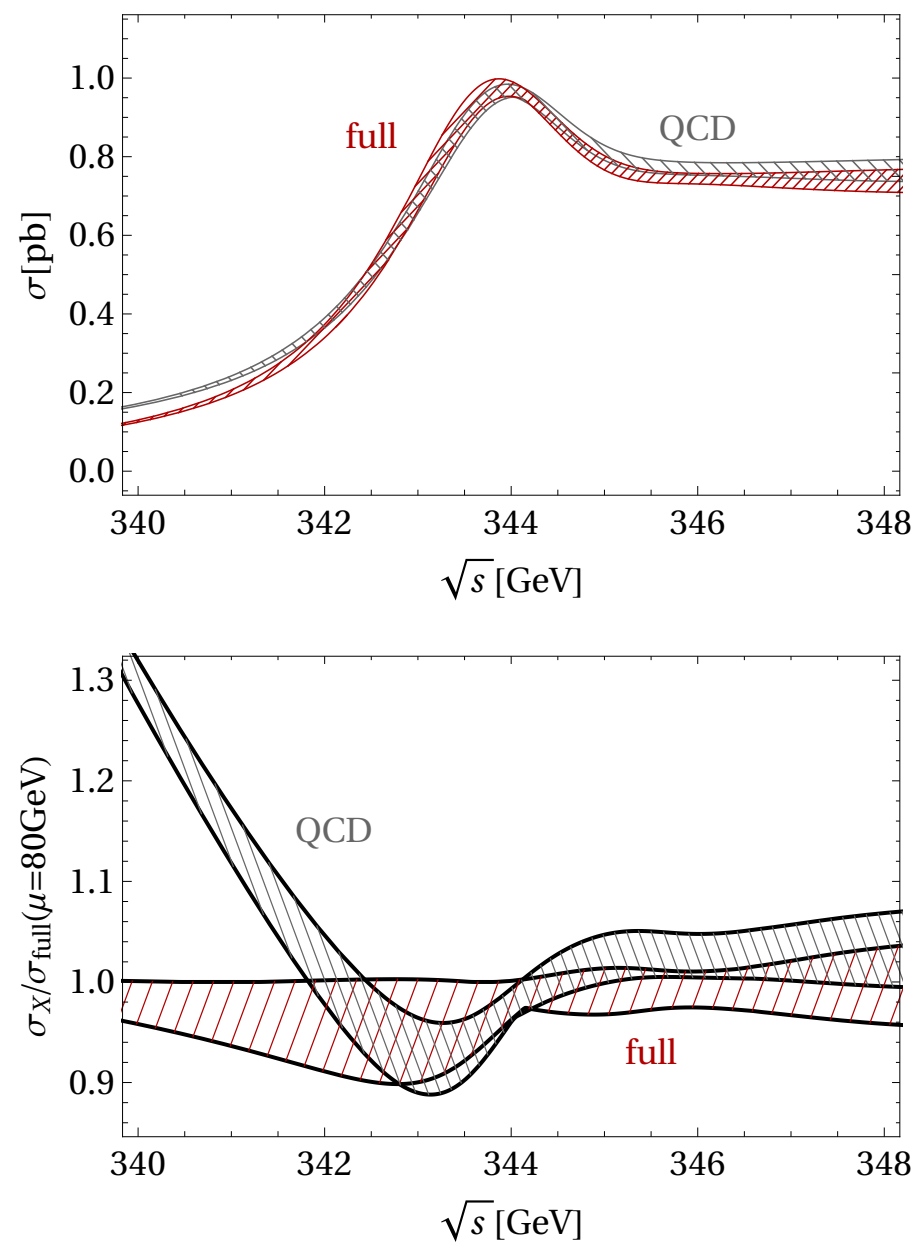

Figure 2: The pure QCD (gray) and full (red) prediction for the total cross section near threshold including the uncertainty from variation of the renormalization scale between 50 and $350 \mathrm{GeV}$. Shown are the cross section in $\mathrm{pb}$ (top) and normalized to the full cross section for the central scale $\mu_{r}=80 \mathrm{GeV}$ (bottom).

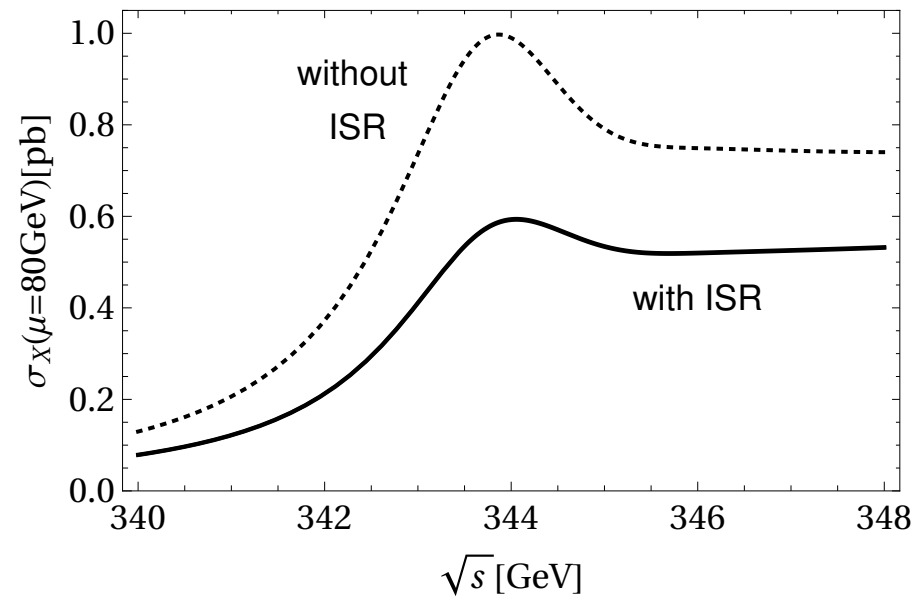

Figure 3: The effect of initial state radiation on the cross section. 
description of ISR which is crucial for precision physics at a future lepton collider. We also note that when electromagnetic initial-state corrections are accounted for at NNLO, the definition of the ISR convolution is factorization-scheme dependent and one can no longer use a phenomenological convolution as is often done in experimental studies without reference to the factorization scheme defining the cross section without ISR.

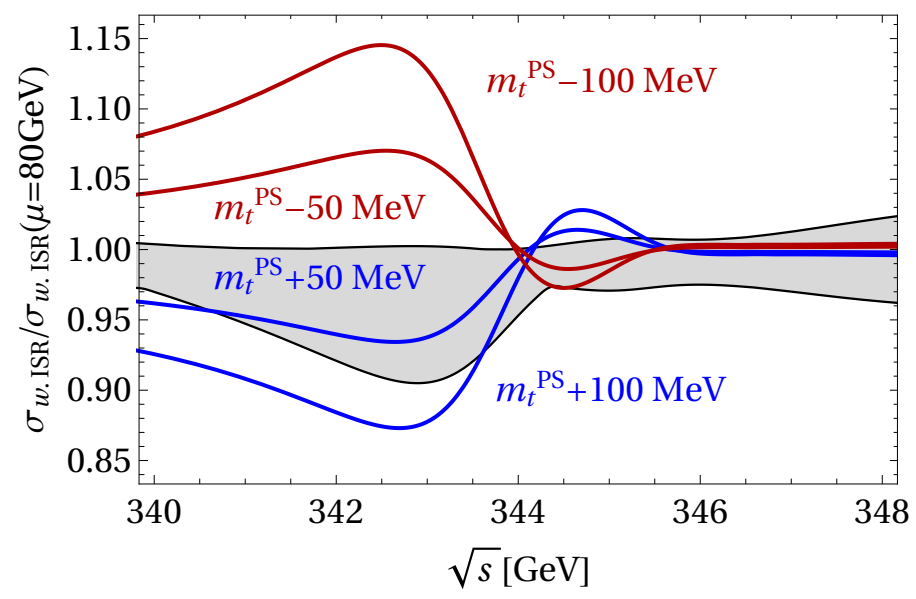

Figure 4: The effect of a variation of the input value of the top-quark mass on the prediction for the cross section. The scale uncertainty band of the full cross section is shown for comparison. The predictions include ISR effects with the accuracy described in Section 3.

The sensitivity of the cross section to the top-quark mass can be estimated by comparing the effects of a variation in the input value to the theoretical uncertainty as shown in Figure 4. To a good approximation a shift $\delta m_{t}$ in the value of the top-quark mass moves the shape of the cross section to the right by $2 \delta m_{t}$. The region where the slope is large is most sensitive to this shift. Comparing the effect to the scale uncertainty we expect that the theoretical uncertainty is of the order of $\pm 40 \mathrm{MeV}$. This estimate is in agreement with the results of a more realistic simulation [2], which takes into account the luminosity spectrum and the properties of the detector. Including statistical uncertainties and experimental systematics, the total uncertainty is expected to be about $\pm 50 \mathrm{MeV}$.

\section{Conclusions}

We presented results of a computation of the complete NNLO non-resonant and electroweak corrections to the total inclusive $b \bar{b} W^{+} W^{-} X$ production cross section in $e^{+} e^{-}$collisions near the top-quark pair production threshold [8]. The contributions are important both conceptually, since they are required to cancel left-over divergences in the pure QCD cross section, as well as phenomenologically, since they lead to sizeable modifications of the cross section. Together with the NNNLO QCD [3, 19] and Higgs [7] contributions, the high precision of the theory predictions allows a determination of the top-quark mass in a well-defined scheme with an uncertainty of about $50 \mathrm{MeV}$ at a future lepton collider.

Last but not least, we note that the EFT techniques developed for these calculations can also be used in other contexts. For instance, the understanding of the behaviour of the $g g \rightarrow H H$ amplitude 
near the top-pair production threshold has recently been used [20] to reconstruct the top-quark mass dependence of the two-loop amplitude [21,22] with high accuracy and the approach used there can also be applied at higher orders and for similar processes.

\section{References}

[1] M. Beneke, Phys. Lett. B 434 (1998) 115 [hep-ph/9804241].

[2] F. Simon, PoS ICHEP 2016 (2017) 872 [arXiv:1611.03399 [hep-ex]].

[3] M. Beneke, Y. Kiyo, P. Marquard, A. Penin, J. Piclum and M. Steinhauser, Phys. Rev. Lett. 115 (2015) no.19, 192001 [arXiv:1506.06864 [hep-ph]].

[4] A. Pineda and J. Soto, Nucl. Phys. Proc. Suppl. 64 (1998) 428 [hep-ph/9707481].

[5] M. Beneke, Y. Kiyo and K. Schuller, arXiv:1312.4791 [hep-ph].

[6] M. Beneke, B. Jantzen and P. Ruiz-Femenia, Nucl. Phys. B 840 (2010) 186 [arXiv:1004.2188 [hep-ph]].

[7] M. Beneke, A. Maier, J. Piclum and T. Rauh, Nucl. Phys. B 899 (2015) 180 [arXiv:1506.06865 [hep-ph]].

[8] M. Beneke, A. Maier, T. Rauh and P. Ruiz-Femenía, to appear.

[9] M. Beneke, A. P. Chapovsky, A. Signer and G. Zanderighi, Phys. Rev. Lett. 93 (2004) 011602 [hep-ph/0312331].

[10] M. Beneke, A. P. Chapovsky, A. Signer and G. Zanderighi, Nucl. Phys. B 686 (2004) 205 [hep-ph/0401002].

[11] M. Beneke and V. A. Smirnov, Nucl. Phys. B 522 (1998) 321 [hep-ph/9711391].

[12] B. Jantzen, JHEP 1112 (2011) 076 [arXiv:1111.2589 [hep-ph]].

[13] B. Jantzen and P. Ruiz-Femenía, Phys. Rev. D 88 (2013) no.5, 054011 [arXiv:1307.4337 [hep-ph]].

[14] J. Alwall et al., JHEP 1407 (2014) 079 [arXiv:1405.0301 [hep-ph]].

[15] A. H. Hoang and C. J. Reißer, Phys. Rev. D 74 (2006) 034002 [hep-ph/0604104].

[16] A. H. Hoang and C. J. Reißer, Phys. Rev. D 71 (2005) 074022 [hep-ph/0412258].

[17] W. Beenakker et al., hep-ph/9602351.

[18] M. Beneke, Y. Kiyo, A. Maier and J. Piclum, Comput. Phys. Commun. 209 (2016) 96 [arXiv:1605.03010 [hep-ph]].

[19] M. Beneke, J. Piclum and T. Rauh, Nucl. Phys. B 880 (2014) 414 [arXiv:1312.4792 [hep-ph]].

[20] R. Gröber, A. Maier and T. Rauh, arXiv:1709.07799 [hep-ph].

[21] S. Borowka, N. Greiner, G. Heinrich, S. P. Jones, M. Kerner, J. Schlenk, U. Schubert and T. Zirke, Phys. Rev. Lett. 117 (2016) no.1, 012001, Erratum: [Phys. Rev. Lett. 117 (2016) no.7, 079901] [arXiv:1604.06447 [hep-ph]].

[22] S. Borowka, N. Greiner, G. Heinrich, S. P. Jones, M. Kerner, J. Schlenk and T. Zirke, JHEP 1610 (2016) 107 [arXiv:1608.04798 [hep-ph]]. 